\title{
NeuroEVs: Characterizing Extracellular Vesicles Generated in the Neural Domain
}

\author{
(1)Christie D. Fowler \\ Department of Neurobiology and Behavior, University of California Irvine, Irvine, California 92697
}

Intercellular communication has recently been shown to occur via transfer of cargo loaded within extracellular vesicles (EVs). Present within all biofluids of the body, EVs can contain various signaling factors, including coding and noncoding RNAs (e.g., mRNA, miRNA, lncRNA, snRNA, tRNA, yRNA), DNA, proteins, and enzymes. Multiple types of cells appear to be capable of releasing EVs, including cancer, stem, epithelial, immune, glial, and neuronal cells. However, the functional impact of these circulating signals among neural networks within the brain has been difficult to establish given the complexity of cellular populations involved in release and uptake, as well as inherent limitations of examining a biofluid. In this brief commentary, we provide an analysis of the conceptual and technical considerations that limit our current understanding of signaling mediated by circulating EVs relative to their impact on neural function.

Key words: exosomes; microvesicles; extracellular RNA signaling; intercellular communication; blood-brain barrier

\section{Introduction}

In recent years, the extracellular vesicle (EV) field has vastly expanded, with findings providing functional implications for both normal physiological and pathological states. Whereas initial investigations assumed that cellular-derived EVs only contained debris, growing evidence has established that these compartments are enriched with a variety of signaling molecules that play crucial roles in many processes (Robbins and Morelli, 2014; Turturici et al., 2014; Budnik et al., 2016; Krämer-Albers and Hill, 2016). Beginning in the 1950s, electron microscopy studies of the CNS suggested the presence of EVs localized within the extracellular space of the brain, with visualization of small vesicles and multivesicular bodies at the cellular-ventricular interface and notation of cytoplasmic organelles containing RNA granules (Maxwell and Pease, 1956; Cupedo, 1977; Ribas, 1977). However, any known significance of these vesicles was not elucidated until more recently. In this Dual Perspectives piece, we first focus on the findings driving our understanding of EV signaling; and thereafter, we discuss technical limitations and considerations that have restricted our ability to ascertain a definitive role for EVs in brain function. However, despite these limitations, an optimistic view of the future is critically maintained as novel technologies and perspectives continue to push boundaries and advance the EV field forward.

\footnotetext{
Received Aug. 21, 2018; revised July 27, 2019; accepted Aug. 15, 2019.

This work was supported by National Institutes of Health Grant DA039658. I thank Dr. John Satterlee (National Institutes of Health) for information related to administering the data coordinating center for the National Institutes of Health Common Fund Extracellular RNA Communication Program.

The authors declare no competing financial interests.

Correspondence should be addressed to Christie D. Fowler at cdfowler@uci.edu.

https://doi.org/10.1523/JNEUROSCI.0146-18.2019

Copyright $\odot 2019$ the authors
}

Function of EVs: brief overview of the field

A groundbreaking study in the immunology field established that dendritic cells release EVs containing tumor antigens, which induce an immune response to suppress tumor growth (Zitvogel et al., 1998). Further studies have documented the role of EVs in mediating some immune-related responses of microglia, B lymphocytes, dendritic cells, T cells, macrophages, and astrocytes (Théry et al., 1999; Denzer et al., 2000; Robbins and Morelli, 2014). In 2007, Valadi et al. (2007) first demonstrated extracellular transfer of RNAs between mast cells. In their investigations, they showed that mast cell-derived EVs contained both mRNAs and miRNAs, and the mRNA from a mouse mast cell was demonstrated to incorporate into a human mast cell line, resulting in expression of the representative protein. Therefore, these studies confirmed both mRNA uptake and subsequent translation by the recipient cell (Valadi et al., 2007), a seminal finding that has greatly advanced the field. More recently, EVs have also been shown to prevent antibody-mediated neutralization of encapsulated viral particles, thereby allowing for the propagation of viral infection (Bello-Morales et al., 2018). Additionally, during an activated neuroimmune response, both microglia and astrocytes release EVs that contain the cytokine interleukin-1 $\beta$ (IL-1 $\beta$ ), which further contributes to immune signaling (Bianco et al., 2005, 2009; Colombo et al., 2018). EVs released from microglia also contain other signaling factors related to the immune response, including major histocompatibility Class II receptors, CD13, TNF, caspase 1, metalloproteinases, tetraspanins, and chaperones (Potolicchio et al., 2005; Robbins and Morelli, 2014; Budnik et al., 2016). Microglia have also been found to propagate the aberrant inflammatory response in multiple sclerosis animal models (Zrzavy et al., 2017), which might in part result from EV cargo uptake into neurons (Prada et al., 2018). Of further inter- 
est, microglia-derived EVs have been implicated in synaptic plasticity and in processes related to Alzheimer's disease pathology (Antonucci et al., 2012; Yuyama and Igarashi, 2017). For example, activation of microglia serotonin receptors results in the release of EVs containing insulin-degrading enzyme along with decreased expression of amyloid- $\beta$ (Glebov et al., 2015); this finding is consistent with the documented correlation in humans between high levels of serotonin and reduced levels of amyloid- $\beta$ in patients (Cirrito et al., 2011).

Within the cancer field, a foundational understanding of EV signaling has also been achieved given the ability to examine isolated cells in culture conditions. Interestingly, oncogenic-related signaling increases the release of EVs from cancer cells, and their EV cargo includes cancer-related proteins, RNA transcripts, and/or genomic DNA; surprisingly, these factors may align with either tumor-promoting or suppressor functions (Yu et al., 2005; Lee et al., 2014; Nakano et al., 2015). Of further note, EVs can mediate metastases in recipient cells (McAllister and Weinberg, 2014; Nogués et al., 2018), and the pattern of integrins on the EV surface appears to determine specificity of target organ trophism (Hoshino et al., 2015). With relevance to the brain, the highly aggressive and malignant glioblastoma cancer cell line has been shown to release EVs that contain miRNAs, mRNAs, histones, and various proteins, which may then affect tumor growth (Skog et al., 2008; Nakano et al., 2015).

EV interactions between neurons and glia have also been identified with in vitro studies. Early seminal studies found that cultured neurons release EVs (Fauré et al., 2006), and subsequent investigations have shown that EVs are released from somatodendritic compartments in response to depolarization in culture conditions (Fauré et al., 2006; Lachenal et al., 2011). EV uptake has been demonstrated to depend on both the EV characteristics and the recipient cell. For example, neuroblastoma-derived EVs appear to be selectively endocytosed by glial cells, whereas EVs released from neurons are preferentially taken up by other neurons in culture (Chivet et al., 2014). These important findings have advanced the theory that cell type-specific interneuronal communication via EVs may mediate information processing within the brain. In addition, oligodendrocytes have also been shown to assist in maintaining neuronal health through EV signaling. Oligodendrocyte-derived EVs alter neuronal firing rate and enhance neuronal viability under conditions of oxygen and glucose deprivation, as assessed in transwell coculture conditions (Frühbeis et al., 2013; Frohlich et al., 2014). NMDA and AMPA glutamate receptors on both neurons and glial cells have been implicated in this EV release, which was demonstrated via pharmacological approaches in culture (Lachenal et al., 2011; Frühbeis et al., 2013; Chivet et al., 2014; Frohlich et al., 2014). Furthermore, EV communication across short distances, such as the synaptic cleft, has been demonstrated as a means of intercellular RNA transfer (Pastuzyn et al., 2018). Finally, EV-mediated signaling can occur between peripheral and neuronal cells. At the neuromuscular junction, retrograde signaling via EV transmission has been demonstrated in Drosophila (Korkut et al., 2013), and hematopoietic-derived EVs have been shown to enter the brain under some inflammatory conditions, which can result in EV miRNA uptake into neurons in mice (Ridder et al., 2014).

Together, the findings to date have provided a basis of knowledge suggesting a possible role for EV signaling in the brain, but it is important to note that these data are mainly based on cell culture conditions, which may not be representative of endogenous mechanisms in a complex biological system, such as the brain or other multicellular organ. Indeed, the functional impact of circulating EVs among neural networks within the brain has remained difficult to establish given the complexity of cellular populations involved in release and uptake, as well as conceptual and technical limitations of the current approaches to analyze EV characteristics and cargo. In the following paragraphs, we discuss these considerations to highlight the current barriers in our ability to draw firm conclusions regarding EV function relative to the dynamic processes occurring in an intact animal.

\section{General EV considerations}

When investigating the potential impact of EV signaling in the brain, it is important to consider the fact that cargos might vary between cells and/or within a given cell population across time. Specifically, many cell types have been shown to release EVs, including immune, tumor, stem, epithelial, glial, and neuronal cells (Kosaka et al., 2010; Turturici et al., 2014; Pastuzyn et al., 2018). The resulting EVs from each cell type may, or may not, differ in content and function. Furthermore, the releasing cell may alter the content/function of EV cargo across time based on varying stimuli and signaling mechanisms. After release into the extracellular space, EVs have been suggested to (1) immediately breakdown to dump contents into the interstitial fluid, (2) travel a short distance to integrate within a cell in close proximity (e.g., across the synapse), or (3) travel a longer distance to integrate into a distant cell population, either through random integration or via a cell-specific targeting motif. Subsequent cellular integration may then lead to changes in target cells via the transfer of proteins, enzymes, DNA, and/or RNA transcripts (e.g., mRNA, miRNA, lncRNA, snRNA, tRNA, yRNA). As noted above, protein and gene expression has been shown to become altered following EV transfer of mRNAs or miRNAs, respectively (Kosaka et al., 2010; Cai et al., 2013; Turturici et al., 2014). Thus far, however, the potential importance of EVs in normal brain function has been difficult to clearly define. For instance, the presence of a vesicle cannot by itself provide evidence of function or relevance for cellular processes since the vesicle might immediately enter the lysosomal degradation pathway after cellular uptake. Moreover, even if EV cargo affects cellular processes, the impact might not be sufficient to exert a measurable effect on cellular function.

Another concern regarding the potential relevance of EV signaling involves the amount of cargo that can be loaded into the vesicle. While this may be of less concern for smaller molecules, such as miRNAs, the transfer of larger molecules, such as mRNAs, within a vesicle may only occur on a 1:1 basis, thus limiting the net impact on a target cell; however, this is admittedly dependent on the number of EVs released, which can be quite substantial. The relative abundance of each type of RNA within a given population of EVs is an additional point of concern; for instance, miRNAs may be less abundant in some populations, leading them to be more challenging to detect when performing measurements based on total RNA in a biological sample (Akers et al., 2013; Chevillet et al., 2014). Furthermore, despite the finding that cells can translate extracellularly derived mRNAs into proteins (Valadi et al., 2007), machinery to further amplify the mRNA has not yet been identified in a recipient cell, indicating that only one protein could conceivably be produced per mRNA transcript. Thus, the impact of a singular protein product within a cell may not result in a significant functional difference. Moreover, any signaling impact would be expected to be limited within a given space and time period. As an example, a released EV may preferentially integrate into an adjacent recipient cell based on "first contact," or may travel a longer distance to achieve integration 
based on "target preference"; alternatively, there may be competition between export out of the brain for subsequent elimination from the body relative to uptake into a target cell, leading to a smaller percentage of EVs actually becoming involved in a functionally significant signaling process. Regarding time-dependent limitations, the impact on the recipient cell would be restricted to the duration of time required for RNA-mediated effects, as it would be unlikely for the target cell to retain the RNA long-term. On the other hand, one must also consider that a larger quantity of cargo does not necessarily imply increased biological relevance. Limited cellular uptake of cargo from the extracellular environment might have the potential to significantly affect neural function, depending on the downstream intracellular processes (Al-Nedawi et al., 2008). For instance, if the RNA cargo targets a transcription factor that modulates multiple genes involved in maintaining cellular function, the resulting effect could lead to changes in a diverse number of cellular processes.

\section{Heterogeneity of EVs}

Heterogeneity of EV populations has become an increasingly intense area of focus since it would be highly desirable to identify biomarkers for EVs released from a specific cell population. However, the large variety of EVs with varying characteristics brings into question what processes can actually be precisely identified in a complex cellular environment, especially given that heterogeneity of EVs exists not only across parent cell populations but also as released from a single-cell type (Zabeo et al., 2017). Although studies have classified EVs based on size, cargo, membrane proteins, targeting motifs, and originating cell population, these categorizations have inherent limitations due to overlapping population characteristics. Several cell types may preferentially release EVs in the $75-150 \mathrm{~nm}$ range or with specific membrane markers, such as CD63 or CD81. Quantification of EVs may also not allow for detection of smaller, but biologically significant, changes in EVs released from the cell population(s) of interest, based on the relative "signal-to-noise ratio" in the sample. Moreover, contamination from other tissues may confound analyses. For example, given that the blood-brain barrier (BBB) may become disrupted during inflammation, stress, or disease states, bidirectional transfer of EVs across this barrier can occur, resulting in both the increased presence of neural-derived EVs in the blood as well as the reciprocal increased presence of peripherally derived EVs in the brain.

\section{EV isolation for characterization}

While recent technological advances have permitted more indepth interrogation of isolated EVs with higher precision (Shao et al., 2018), the ability to characterize centrally derived EV populations from biologically relevant samples remains challenging. One major factor is the large volume (e.g., $\sim 5 \mathrm{ml}$ ) of starting sample required for preferred isolation techniques, such as ultracentrifugation or sucrose-gradient centrifugation. Higher purity with these approaches also comes at a cost of significant sample loss with low recovery. Thus, if the target cargo is of low quantity within the sample or if the sample size is limited, the "goldstandard" isolation methods may not be the most appropriate experimental approach. Ultracentrifugation may also result in damage to the vesicle shape or structure, which would impact subsequent use for further studies (e.g., to determine target cell integration following EV injection in vivo). In addition to centrifugation, other methods, such as immunoprecipitation or antibody-based sorting, can also result in selective enrichment of certain populations of EVs or cargo, leading to biased sampling
(Mateescu et al., 2017). Immunoprecipitation has further been found to allow for extraction of non-EV constituents of the sample (Théry et al., 2018). Moreover, disproportionate representation of EV subpopulation cargo may be amplified with certain techniques, such as biased amplification and detection of RNA transcripts with current RNAseq platforms. However, comparisons across experimental conditions can be performed when factoring in the potential for bias, as all group conditions would have undergone similar processing and analysis, although it should still be acknowledged that a target of interest may not be accurately represented based on these limitations.

The potential for variability both within and across platforms also introduces concern for accurate data interpretation when analyzing multiple samples. A recent study demonstrated variability of EV quantification with nanoparticle tracking when using standardized settings on two different instruments, but this variability was reduced by optimizing settings on an instrumentspecific basis (Vestad et al., 2017). These data highlight the importance of analyzing all samples for a given study under tightly controlled conditions on the same instrument, which represents a significant challenge for longitudinal studies. Furthermore, nanoparticle tracking detects any particle within a given size range; thus, the data may not only be representative of EV populations. Therefore, validation across technical platforms should be incorporated into experimental design as a further means to validate conclusions.

\section{EV cargo quantification}

After isolating EVs, assessment of cargo is often conducted to elucidate changes in EV-specific signaling factors. However, the selection of a reference RNA or protein as a normalization factor has remained a concerning issue for interpretation of data since variable levels of RNAs and proteins are found under baseline conditions. For instance, to quantify protein levels in brain tissue, $\beta$-actin is often used as the reference to adjust for differences in the number of cells collected between subjects; however, such a universally accepted reference has not been consistently ascertained for biofluids. Conceptually, if an increase in the number of EV-encapsulated miRNAs occurs following a stimulus, the most appropriate reference would be a miRNA that is found in high abundance in the biofluid and that is unaltered by the specific experimental stimulus. Without an appropriate reference miRNA, it is unclear as to how to control for differences based on unavoidable variation in sampling between subjects. To address this issue, some investigators have chosen to normalize to total RNA counts, total protein levels, or EV density, but each of these approaches often leads to different data endpoints and, thus, questionable conclusions. With regard to an appropriate singular reference RNA for qRT-PCR quantification, some in the field use U6, but there is evidence that U6 can have variable expression in biofluid samples (Xiang et al., 2014). This has led others to propose averaging multiple putative reference miRNAs together to ascertain an average background level of expression or estimating the total number of EVs in a sample (Akers et al., 2013; Gouin et al., 2017), but these approaches may still lead to biased findings depending on the miRNAs selected or EV quantification method used, respectively.

When changes are found in EV characteristics and/or cargo following an experimental stimulus or between disease conditions in vivo, any identified difference(s) could feasibly be the result of a variety of mechanisms, including cell population of origin, preferential loading of certain cargo, release dynamics, and/or kinetics associated with cell type-specific uptake. Thus, 
interpretation of the relevance of any detected change may be based on speculative assertations, rather than definitive conclusions. By validating in vivo findings with more experimentally controlled in vitro conditions, individual processes can be discretely isolated and assessed with a higher level of confidence. However, established findings in the field should guide in vitro experimental design. For instance, many earlier publications used FBS in cell culture experiments; however, FBS has been found to contain bovine EVs/RNAs that have contaminated results (Wei et al., 2016; Kornilov et al., 2018). Second, the formation of nanotubules can occur in culture conditions, which allow for the transfer of materials between cells; this transfer process could confound investigations seeking to demonstrate EV cellto-cell communication (McCoy-Simandle et al., 2016). Third, strategies to identify EVs via lipophilic dye labeling have been shown to lead to fluorescent dye transfer out of labeled EVs and into other cells (Takov et al., 2017), resulting in incorrect assessment of EV number and target cell integration. As the field continues to evolve, a set of guidelines to establish "minimal information for studies of extracellular vesicles" was put forth to provide specific criteria to enhance rigor for EV studies and to provide a foundation for comparisons across the field (Lötvall et al., 2014; Théry et al., 2018).

\section{Further considerations for brain-derived circulating EVs}

Various approaches have been used to assess and characterize EVs selectively derived from cells in the brain. For instance, CSF has been collected and analyzed to examine EVs localized within the intracerebral space. However, it is often challenging to obtain a pure sample because techniques to access the interstitial fluid of the brain or spinal cord can induce puncture of blood vesicles, resulting in blood-derived EV contamination. To overcome this issue, chronic indwelling implants may be used to collect small samples over time. Unfortunately, in rodents, the size of the sample continues to be a limiting factor for further analysis because many EV assays require milliliters of fluid, whereas only a few microliters may be obtained at a time with this method. Alternatively, CSF may be obtained from the cisterna magna after carefully dissecting and removing muscle covering the outer membrane at the base of the skull; when performed precisely, this method allows for collection of pure CSF without blood contamination. While relatively larger samples can be obtained with this technique in rodents, it is a terminal procedure that still only yields microliter-range sample sizes (e.g., $\sim 10-15 \mu \mathrm{l}$ from mice, $\sim 100-200 \mu \mathrm{l}$ from rats). In humans, ventriculoperitoneal shunts may be implanted under pathological conditions to allow for CSF collection over time, but comparison control samples to account for changes with the disease state are more difficult to obtain due to safety issues based on the invasive nature of the procedure for healthy subjects. Moreover, the location of sample collection has been shown to lead to differences in EV RNA content (Akers et al., 2013), thus imposing additional challenges to ensure experimental rigor.

Extraction methods have also been developed to derive EVs from the extracellular (nonventricular) space surrounding neurons by dissecting brain tissue and processing to isolate EV populations (Gallart-Palau et al., 2016; Vella et al., 2017). This technical approach may seek to limit intracellular contamination, but damage to cell membranes with dissection is unavoidable and thus induces the release of intracellular vesicles and/or leads to the artificial creation of vesicles (e.g., synaptosome-like vesicles). Consequently, enrichment, rather than purification, is more specifically obtained with current approaches (Vella et al.,
2017), a consideration that must be addressed when interpreting experimental findings.

While EVs in the brain are thought to mainly contribute to intercellular neural signaling, it is likely that either (1) excess EVs are released, leading to the need for filtration and elimination from the body, and/or (2) neural-derived EVs may be incorporated into peripheral cells to modulate their function. For this to occur, EVs would have to pass through the BBB. While the exact mechanisms of active or passive transport across this barrier are largely unknown, there is evidence that this does indeed occur. For instance, intravenously administered EVs have been shown to enter into the neural parenchyma (Alvarez-Erviti et al., 2011); and conversely, altered expression of EVs is found in blood samples from patients with CNS neurological disease (Burgos et al., 2014). Together, these findings provide evidence for the reciprocal passage of EVs between the brain and blood. However, the selective isolation of neural-derived EVs in peripheral biofluids is currently not feasible given the lack of a neuronspecific biomarker. Indeed, proposed "neuron-derived" markers (e.g., L1CAM or NCAM) are not necessarily neuron specific. Cross-referencing these markers to protein expression patterns in peripheral organs (e.g., via databases, such as www.proteinatlas.org) provides evidence that EVs with these markers could have originated from peripheral tissues.

Since the identification and detection of EVs in the urine or blood hold promise to elucidate biomarkers of neural signaling or pathological states, studies are being conducted to establish a correlation between brain state and peripheral EV population characteristics. However, collection/processing of urine or blood can introduce contaminating factors, such as bacteria or an activated immune response, respectively. If urine is not sterilefiltered a short period of time after collection, bacteria-derived EVs may be introduced into the sample and thereby contaminate analyses. When handling blood, the extraction and processing can lead to platelet contamination and/or immune cells can be artificially induced to release EVs that were not present in the fluid at the time of blood collection (Mitchell et al., 2016). Furthermore, consistent processing protocols need to be implemented to allow for confidence in the findings with comparisons of samples. For instance, after collection of a large volume of CSF from a human, samples may undergo repeated freeze-thaw cycles each time a smaller amount is extracted to obtain multiple data points, such as to conduct various technical analyses (e.g., EM, EV quantification, RNA analysis, protein analysis, etc.) or to compile repeated assessments for a longitudinal study. However, repeated freeze-thaw cycles have been shown to be especially detrimental to sample integrity (Yuana et al., 2015; Akers et al., 2016).

The dynamics associated with EV signaling also introduce question for our ability to derive firm conclusions based on assessment of EV number and/or cargo in a biological sample. For example, an increase in the number of EVs is not necessarily indicative of increased parental cell EV release. Rather, it may be attributable to a decrease in EV uptake into target cells. Conversely, a decrease in EV density could be indicative of decreased release from parental cells, increased uptake by target cells, and/or increased export out of the brain. All of these factors are influenced by sample collection time point relative to the experimental manipulation, as well as largely undefined timedependent factors, such as those involved in cell-specific EV biogenesis, release, and uptake. 


\section{EV delivery into the brain}

In addition to the assessment of EVs derived from the neural domain, the generation of EVs for directed delivery of therapeutic molecules or drug compounds into the brain is a quickly advancing area within the field (Lakhal and Wood, 2011; GarcíaManrique et al., 2018; Zhu et al., 2018). In support of this therapeutic approach, systemic injections of EVs derived from mesenchymal stromal cells were found to enhance neural recovery after ischemic stroke in rats (Xin et al., 2013). Such a strategy has tremendous therapeutic potential if cell type-specific integration can be achieved via external targeting motifs. However, skepticism is present for whether this approach can be successfully applied in a clinically relevant setting. First, given that EVs are only $\sim 40 \mathrm{~nm}$ to $1 \mu \mathrm{m}$ in size, the type and amount of cargo able to be loaded are limited; and therefore, therapeutic approaches may need to be in the nanoscale range. To date, engineered EVs have successfully been loaded with $70-250 \mathrm{kDa}$ molecules, although the current methods have had low success in generating a large yield of viable EVs (Antimisiaris et al., 2018). Second, the targeted delivery method must be validated for selectivity and off-target effects. For example, because peripheral injection for subsequent integration across the BBB would be preferred (e.g., as opposed to intracerebroventricular injection), the quantity of EVs injected will likely need to be tightly controlled to prevent adverse effects resulting from EV accumulation and/or breakdown in peripheral organ systems. Further, a large number of injected EVs will be required to obtain adequate infiltration into the brain, until mechanisms to promote passage through the $\mathrm{BBB}$ are more clearly defined and exploited.

\section{Future outlook and perspectives to advance the EV field}

To achieve rigor and reproducibility in the field, validation of techniques to obtain, analyze, and interpret EV data from the extracellular environment will need to continue evolving as varying types of samples are targeted for experimentation. Biased, but replicable, assessments may be an appropriate starting point for some studies with inherent technical limitations. Cross-platform validation should also be incorporated to support conclusions, when possible. Another goal is to be better able to obtain multiple data points from the same sample, such as density and size quantification paired with subsequent analysis of cargo content, or alternatively, the ability to maintain EV integrity for injection in vivo following various isolation methods. Along these lines, the ability to probe for a variety of EV content, including multiple RNA species, RNA binding proteins, DNA, enzymes, and other proteins, in a cell type- and stimulus-specific manner will be important to fully understand the various functions of this type of extracellular communication. Of note, the National Institutes of Health Common Fund Extracellular RNA Communication Program has developed an exRNA online resource portal (www. exrna.org), which provides searchable datasets for different biofluids derived from individuals characterized as "normal" or diagnosed with disease, a software pipeline to analyze EV datasets, metadata standards to allow informative labeling of EV datasets, protocols to standardize EV research, and EV-relevant resources, such as plasmids, mice, and libraries. Open source depositing of large datasets will continue to assist in defining specificity of cargo and/or targeting motifs, especially when based on cell type of origin and/or disease state, although this is dependent on continued support to maintain such efforts (e.g., www. exocarta.org and www.microvesicles.org).

The field will also need to obtain a more systematic understanding of the biogenesis, packaging, release, and uptake time courses for EVs derived from individual cell types. For instance, future studies will need to define the relative time necessary to do the following: (1) initiate intracellular signaling cascades to produce specific EV cargo, such as miRNAs, mRNAs, IncRNAs proteins, enzymes, etc., (2) generate EV membranes as either multivesicular bodies or via membrane blebbing, (3) shuttle signaling factors to the EV location, (4) load EVs, (5) release EVs into the extracellular environment, (6) uptake EVs by the recipient cell, (7) degrade the EV membrane and process the EV contents, and (8) exert an impact on the recipient cell's function. While several of these processes are beginning to become elucidated, further validation will need to occur across cell types and in vivo. Indeed, the ongoing development of transgenic mouse lines to detect EVs released from target cell subpopulations has the potential to lead to significant advances. Along these lines, current National Institutes of Health funding initiatives are focused on the development of novel technologies to catalyze discoveries and further expand our understanding of EV-based communication.

In conclusion, EVs represent an exciting new direction for the neuroscience field. As the field progresses, new discoveries will further establish a greater understanding of these signaling compartments and their cargo, with the potential for broad significance and inferences across neuroscience subfields. For instance, it will truly be exciting to determine whether EV signaling mechanisms underlie brain development, homeostatic function, sensory processing, motivated behaviors, and cognitive function, as a few possibilities. Ultimately, the canonical characterization of neural communication methods will likely have to be expanded beyond electrical, chemical, and hormonal mechanisms to now include EV signaling mechanisms.

\section{References}

Akers JC, Ramakrishnan V, Kim R, Skog J, Nakano I, Pingle S, Kalinina J, Hua W, Kesari S, Mao Y, Breakefield XO, Hochberg FH, Van Meir EG, Carter BS, Chen CC (2013) MiR-21 in the extracellular vesicles (EVs) of cerebrospinal fluid (CSF): a platform for glioblastoma biomarker development. PLoS One 8:e78115.

Akers JC, Ramakrishnan V, Yang I, Hua W, Mao Y, Carter BS, Chen CC (2016) Optimizing preservation of extracellular vesicular miRNAs derived from clinical cerebrospinal fluid. Cancer Biomark 17:125-132.

Al-Nedawi K, Meehan B, Micallef J, Lhotak V, May L, Guha A, Rak J (2008) Intercellular transfer of the oncogenic receptor EGFRvIII by microvesicles derived from tumour cells. Nat Cell Biol 10:619-624.

Alvarez-Erviti L, Seow Y, Yin H, Betts C, Lakhal S, Wood MJ (2011) Delivery of siRNA to the mouse brain by systemic injection of targeted exosomes. Nat Biotechnol 29:341-345.

Antimisiaris SG, Mourtas S, Marazioti A (2018) Exosomes and exosomeinspired vesicles for targeted drug delivery. Pharmaceutics 10:e218.

Antonucci F, Turola E, Riganti L, Caleo M, Gabrielli M, Perrotta C, Novellino L, Clementi E, Giussani P, Viani P, Matteoli M, Verderio C (2012) Microvesicles released from microglia stimulate synaptic activity via enhanced sphingolipid metabolism. EMBO J 31:1231-1240.

Bello-Morales R, Praena B, de la Nuez C, Rejas MT, Guerra M, Galán-Ganga M, Izquierdo M, Calvo V, Krummenacher C, López-Guerrero JA (2018) Role of microvesicles in the spread of Herpes simplex virus 1 in oligodendrocytic cells. J Virol 92:e00088.

Bianco F, Pravettoni E, Colombo A, Schenk U, Möller T, Matteoli M, Verderio C (2005) Astrocyte-derived ATP induces vesicle shedding and IL-1 beta release from microglia. J Immunol 174:7268-7277.

Bianco F, Perrotta C, Novellino L, Francolini M, Riganti L, Menna E, Saglietti L, Schuchman EH, Furlan R, Clementi E, Matteoli M, Verderio C (2009) Acid sphingomyelinase activity triggers microparticle release from glial cells. EMBO J 28:1043-1054.

Budnik V, Ruiz-Cañada C, Wendler F (2016) Extracellular vesicles round off communication in the nervous system. Nat Rev Neurosci 17:160-172.

Burgos K, Malenica I, Metpally R, Courtright A, Rakela B, Beach T, Shill H, Adler C, Sabbagh M, Villa S, Tembe W, Craig D, Van Keuren-Jensen K 
(2014) Profiles of extracellular miRNA in cerebrospinal fluid and serum from patients with Alzheimer's and Parkinson's diseases correlate with disease status and features of pathology. PLoS One 9:e94839.

Cai J, Han Y, Ren H, Chen C, He D, Zhou L, Eisner GM, Asico LD, Jose PA, Zeng C (2013) Extracellular vesicle-mediated transfer of donor genomic DNA to recipient cells is a novel mechanism for genetic influence between cells. J Mol Cell Biol 5:227-238.

Chevillet JR, Kang Q, Ruf IK, Briggs HA, Vojtech LN, Hughes SM, Cheng HH, Arroyo JD, Meredith EK, Gallichotte EN, Pogosova-Agadjanyan EL, Morrissey C, Stirewalt DL, Hladik F, Yu EY, Higano CS, Tewari M (2014) Quantitative and stoichiometric analysis of the microRNA content of exosomes. Proc Natl Acad Sci U S A 111:14888-14893.

Chivet M, Javalet C, Laulagnier K, Blot B, Hemming FJ, Sadoul R (2014) Exosomes secreted by cortical neurons upon glutamatergic synapse activation specifically interact with neurons. J Extracell Vesicles 3:24722.

Cirrito JR, Disabato BM, Restivo JL, Verges DK, Goebel WD, Sathyan A, Hayreh D, D’Angelo G, Benzinger T, Yoon H, Kim J, Morris JC, Mintun MA, Sheline YI (2011) Serotonin signaling is associated with lower amyloid-beta levels and plaques in transgenic mice and humans. Proc Natl Acad Sci U S A 108:14968-14973.

Colombo F, Bastoni M, Nigro A, Podini P, Finardi A, Casella G, Ramesh M, Farina C, Verderio C, Furlan R (2018) Cytokines stimulate the release of microvesicles from myeloid cells independently from the $\mathrm{P} 2 \mathrm{X} 7$ receptor/ acid sphingomyelinase pathway. Front Immunol 9:204.

Cupedo RN (1977) The surface ultrastructure of the habenular complex of the rat. Anat Embryol (Berl) 152:43-64.

Denzer K, van Eijk M, Kleijmeer MJ, Jakobson E, de Groot C, Geuze HJ (2000) Follicular dendritic cells carry MHC class II-expressing microvesicles at their surface. J Immunol 165:1259-1265.

Fauré J, Lachenal G, Court M, Hirrlinger J, Chatellard-Causse C, Blot B, Grange J, Schoehn G, Goldberg Y, Boyer V, Kirchhoff F, Raposo G, Garin J, Sadoul R (2006) Exosomes are released by cultured cortical neurones. Mol Cell Neurosci 31:642-648.

Frohlich D, Kuo WP, Frühbeis C, Sun JJ, Zehendner CM, Luhmann HJ, Pinto S, Toedling J, Trotter J, Krämer-Albers EM (2014) Multifaceted effects of oligodendroglial exosomes on neurons: impact on neuronal firing rate, signal transduction and gene regulation. Philos Trans R Soc Lond B Biol Sci 369:pii20130510.

Frühbeis C, Fröhlich D, Kuo WP, Amphornrat J, Thilemann S, Saab AS, Kirchhoff F, Möbius W, Goebbels S, Nave KA, Schneider A, Simons M, Klugmann M, Trotter J, Krämer-Albers EM (2013) Neurotransmittertriggered transfer of exosomes mediates oligodendrocyte-neuron communication. PLoS Biol 11:e1001604.

Gallart-Palau X, Serra A, Sze SK (2016) Enrichment of extracellular vesicles from tissues of the central nervous system by PROSPR. Mol Neurodegener 11:41.

García-Manrique P, Matos M, Gutiérrez G, Pazos C, Blanco-López MC (2018) Therapeutic biomaterials based on extracellular vesicles: classification of bio-engineering and mimetic preparation routes. J Extracell Vesicles 7:1422676.

Glebov K, Löchner M, Jabs R, Lau T, Merkel O, Schloss P, Steinhäuser C, Walter J (2015) Serotonin stimulates secretion of exosomes from microglia cells. Glia 63:626-634.

Gouin K, Peck K, Antes T, Johnson JL, Li C, Vaturi SD, Middleton R, de Couto G, Walravens AS, Rodriguez-Borlado L, Smith RR, Marbán L, Marbán E, Ibrahim AG (2017) A comprehensive method for identification of suitable reference genes in extracellular vesicles. J Extracell Vesicles 6:1347019.

Hoshino A, Costa-Silva B, Shen TL, Rodrigues G, Hashimoto A, Tesic Mark M, Molina H, Kohsaka S, Di Giannatale A, Ceder S, Singh S, Williams C, Soplop N, Uryu K, Pharmer L, King T, Bojmar L, Davies AE, Ararso Y, Zhang T, et al. (2015) Tumour exosome integrins determine organotropic metastasis. Nature 527:329-335.

Korkut C, Li Y, Koles K, Brewer C, Ashley J, Yoshihara M, Budnik V (2013) Regulation of postsynaptic retrograde signaling by presynaptic exosome release. Neuron 77:1039-1046.

Kornilov R, Puhka M, Mannerström B, Hiidenmaa H, Peltoniemi H, Siljander P, Seppänen-Kaijansinkko R, Kaur S (2018) Efficient ultrafiltration-based protocol to deplete extracellular vesicles from fetal bovine serum. J Extracell Vesicles 7:1422674.

Kosaka N, Iguchi H, Yoshioka Y, Takeshita F, Matsuki Y, Ochiya T (2010)
Secretory mechanisms and intercellular transfer of microRNAs in living cells. J Biol Chem 285:17442-17452.

Krämer-Albers EM, Hill AF (2016) Extracellular vesicles: interneural shuttles of complex messages. Curr Opin Neurobiol 39:101-107.

Lachenal G, Pernet-Gallay K, Chivet M, Hemming FJ, Belly A, Bodon G, Blot B, Haase G, Goldberg Y, Sadoul R (2011) Release of exosomes from differentiated neurons and its regulation by synaptic glutamatergic activity. Mol Cell Neurosci 46:409-418.

Lakhal S, Wood MJ (2011) Exosome nanotechnology: an emerging paradigm shift in drug delivery. Exploitation of exosome nanovesicles for systemic in vivo delivery of RNAi heralds new horizons for drug delivery across biological barriers. Bioessays 33:737-741.

Lee TH, Chennakrishnaiah S, Audemard E, Montermini L, Meehan B, Rak J (2014) Oncogenic ras-driven cancer cell vesiculation leads to emission of double-stranded DNA capable of interacting with target cells. Biochem Biophys Res Commun 451:295-301.

Lötvall J, Hill AF, Hochberg F, Buzás EI, Di Vizio D, Gardiner C, Gho YS, Kurochkin IV, Mathivanan S, Quesenberry P, Sahoo S, Tahara H, Wauben MH, Witwer KW, Théry C (2014) Minimal experimental requirements for definition of extracellular vesicles and their functions: a position statement from the international society for extracellular vesicles. J Extracell Vesicles 3:26913.

Mateescu B, Kowal EJ, van Balkom BW, Bartel S, Bhattacharyya SN, Buzás EI, Buck AH, de Candia P, Chow FW, Das S, Driedonks TA, FernándezMessina L, Haderk F, Hill AF, Jones JC, Van Keuren-Jensen KR, Lai CP, Lässer C, Liegro ID, Lunavat TR, et al. (2017) Obstacles and opportunities in the functional analysis of extracellular vesicle RNA: an ISEV position paper. J Extracell Vesicles 6:1286095.

Maxwell DS, Pease DC (1956) The electron microscopy of the choroid plexus. J Biophys Biochem Cytol 2:467-474.

McAllister SS, Weinberg RA (2014) The tumour-induced systemic environment as a critical regulator of cancer progression and metastasis. Nat Cell Biol 16:717-727.

McCoy-Simandle K, Hanna SJ, Cox D (2016) Exosomes and nanotubes: control of immune cell communication. Int J Biochem Cell Biol 71:4454.

Mitchell AJ, Gray WD, Hayek SS, Ko YA, Thomas S, Rooney K, Awad M, Roback JD, Quyyumi A, Searles CD (2016) Platelets confound the measurement of extracellular miRNA in archived plasma. Sci Rep 6:32651.

Nakano I, Garnier D, Minata M, Rak J (2015) Extracellular vesicles in the biology of brain tumour stem cells: implications for inter-cellular communication, therapy and biomarker development. Semin Cell Dev Biol 40:17-26.

Nogués L, Benito-Martin A, Hergueta-Redondo M, Peinado H (2018) The influence of tumour-derived extracellular vesicles on local and distal metastatic dissemination. Mol Aspects Med 60:15-26.

Pastuzyn ED, Day CE, Kearns RB, Kyrke-Smith M, Taibi AV, McCormick J, Yoder N, Belnap DM, Erlendsson S, Morado DR, Briggs JA, Feschotte C, Shepherd JD (2018) The neuronal gene arc encodes a repurposed retrotransposon gag protein that mediates intercellular RNA transfer. Cell 172:275-288.e18.

Potolicchio I, Carven GJ, Xu X, Stipp C, Riese RJ, Stern LJ, Santambrogio L (2005) Proteomic analysis of microglia-derived exosomes: metabolic role of the aminopeptidase CD13 in neuropeptide catabolism. J Immunol 175:2237-2243.

Prada I, Gabrielli M, Turola E, Iorio A, D’Arrigo G, Parolisi R, De Luca M, Pacifici M, Bastoni M, Lombardi M, Legname G, Cojoc D, Buffo A, Furlan R, Peruzzi F, Verderio C (2018) Glia-to-neuron transfer of miRNAs via extracellular vesicles: a new mechanism underlying inflammationinduced synaptic alterations. Acta Neuropathol 135:529-550.

Ribas JL (1977) Morphological evidence for a possible functional role of supra-ependymal nerves on ependyma. Brain Res 125:362-368.

Ridder K, Keller S, Dams M, Rupp AK, Schlaudraff J, Del Turco D, Starmann J, Macas J, Karpova D, Devraj K, Depboylu C, Landfried B, Arnold B, Plate KH, Höglinger G, Sültmann H, Altevogt P, Momma S (2014) Extracellular vesicle-mediated transfer of genetic information between the hematopoietic system and the brain in response to inflammation. PLoS Biol 12:e1001874.

Robbins PD, Morelli AE (2014) Regulation of immune responses by extracellular vesicles. Nat Rev Immunol 14:195-208.

Shao H, Im H, Castro CM, Breakefield X, Weissleder R, Lee H (2018) New 
technologies for analysis of extracellular vesicles. Chem Rev 118:1917-1950.

Skog J, Würdinger T, van Rijn S, Meijer DH, Gainche L, Sena-Esteves M, Curry WT Jr, Carter BS, Krichevsky AM, Breakefield XO (2008) Glioblastoma microvesicles transport RNA and proteins that promote tumour growth and provide diagnostic biomarkers. Nat Cell Biol 10:1470-1476.

Takov K, Yellon DM, Davidson SM (2017) Confounding factors in vesicle uptake studies using fluorescent lipophilic membrane dyes. J Extracell Vesicles 6:1388731.

Théry C, Regnault A, Garin J, Wolfers J, Zitvogel L, Ricciardi-Castagnoli P, Raposo G, Amigorena S (1999) Molecular characterization of dendritic cell-derived exosomes: selective accumulation of the heat shock protein hsc73. J Cell Biol 147:599-610.

Théry C, Witwer KW, Aikawa E, Alcaraz MJ, Anderson JD, Andriantsitohaina R, Antoniou A, Arab T, Archer F, Atkin-Smith GK, Ayre DC, Bach JM, Bachurski D, Baharvand H, Balaj L, Baldacchino S, Bauer NN, Baxter AA, Bebawy M, Beckham C, et al. (2018) Minimal information for studies of extracellular vesicles 2018 (MISEV2018): a position statement of the International Society for Extracellular Vesicles and update of the MISEV2014 guidelines. J Extracell Vesicles 7:1535750.

Turturici G, Tinnirello R, Sconzo G, Geraci F (2014) Extracellular membrane vesicles as a mechanism of cell-to-cell communication: advantages and disadvantages. Am J Physiol Cell Physiol 306:C621-C633.

Valadi H, Ekström K, Bossios A, Sjöstrand M, Lee JJ, Lötvall JO (2007) Exosome-mediated transfer of mRNAs and microRNAs is a novel mechanism of genetic exchange between cells. Nat Cell Biol 9:654-659.

Vella LJ, Scicluna BJ, Cheng L, Bawden EG, Masters CL, Ang CS, Willamson N, McLean C, Barnham KJ, Hill AF (2017) A rigorous method to enrich for exosomes from brain tissue. J Extracell Vesicles 6:1348885.

Vestad B, Llorente A, Neurauter A, Phuyal S, Kierulf B, Kierulf P, Skotland T, Sandvig K, Haug KB, Øvstebo R (2017) Size and concentration analyses of extracellular vesicles by nanoparticle tracking analysis: a variation study. J Extracell Vesicles 6:1344087.
Wei Z, Batagov AO, Carter DR, Krichevsky AM (2016) Fetal bovine serum RNA interferes with the cell culture derived extracellular RNA. Sci Rep 6:31175.

Xiang M, Zeng Y, Yang R, Xu H, Chen Z, Zhong J, Xie H, Xu Y, Zeng X (2014) U6 is not a suitable endogenous control for the quantification of circulating microRNAs. Biochem Biophys Res Commun 454:210-214.

Xin H, Li Y, Cui Y, Yang JJ, Zhang ZG, Chopp M (2013) Systemic administration of exosomes released from mesenchymal stromal cells promote functional recovery and neurovascular plasticity after stroke in rats. J Cereb Blood Flow Metab 33:1711-1715.

Yu JL, May L, Lhotak V, Shahrzad S, Shirasawa S, Weitz JI, Coomber BL, Mackman N, Rak JW (2005) Oncogenic events regulate tissue factor expression in colorectal cancer cells: implications for tumor progression and angiogenesis. Blood 105:1734-1741.

Yuana Y, Böing AN, Grootemaat AE, van der Pol E, Hau CM, Cizmar P, Buhr E, Sturk A, Nieuwland R (2015) Handling and storage of human body fluids for analysis of extracellular vesicles. J Extracell Vesicles 4:29260.

Yuyama K, Igarashi Y (2017) Exosomes as carriers of Alzheimer's amyloidss. Front Neurosci 11:229.

Zabeo D, Cvjetkovic A, Lässer C, Schorb M, Lötvall J, Höög JL (2017) Exosomes purified from a single cell type have diverse morphology. J Extracell Vesicles 6:1329476.

Zhu L, Oh JM, Gangadaran P, Kalimuthu S, Baek SH, Jeong SY, Lee SW, Lee J, Ahn BC (2018) Targeting and therapy of glioblastoma in a mouse model using exosomes derived from natural killer cells. Front Immunol 9:824.

Zitvogel L, Regnault A, Lozier A, Wolfers J, Flament C, Tenza D, RicciardiCastagnoli P, Raposo G, Amigorena S (1998) Eradication of established murine tumors using a novel cell-free vaccine: dendritic cell-derived exosomes. Nat Med 4:594-600.

Zrzavy T, Hametner S, Wimmer I, Butovsky O, Weiner HL, Lassmann H (2017) Loss of 'homeostatic' microglia and patterns of their activation in active multiple sclerosis. Brain 140:1900-1913. 